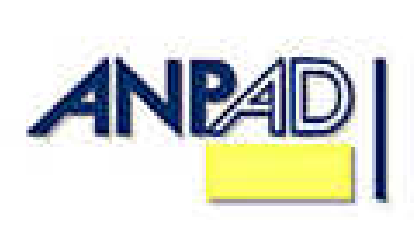

Disponível em

http://www.anpad.org.br/rac

RAC, Curitiba, v. 14, n. 1, art. 2, pp. 20-39, Jan./Fev. 2010

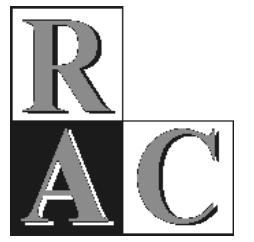

\title{
Direcionadores na Difusão da Estratégia de Postponement: Casos de Empresas Brasileiras
}

Pointers in the Spread of Postponement Strategy: Cases of Brazilian Companies

Mauro Sampaio *

Doutor em Administração de Empresas pela FGV. Professor do POI/EAESP/FGV, São Paulo/SP, Brasil.

João Mario Csillag

Doutor em Administração de Empresas pela FGV. Professor do POI/EAESP/FGV, São Paulo/SP, Brasil.

\footnotetext{
* Endereço: Mauro Sampaio

Fundação Getulio Vargas EAESP, Rua Itapeva, 474, $8^{\circ}$ andar, Bela Vista, São Paulo/SP, 01332-000. E-mail: mauro.sampaio@fgv.br
}

Copyright (C) 2010 RAC. Todos os direitos, inclusive de tradução, são reservados. É permitido citar parte de artigos sem autorização prévia desde que seja identificada a fonte. 


\title{
RESUMO
}

O propósito deste artigo foi investigar a difusão do postponement no ambiente de negócios brasileiro e identificar os direcionadores de sua implementação. Este estudo utilizou a metodologia de multicasos para avaliar um fenômeno muito conhecido na literatura, mas raro no mundo empresarial. Resultados sugerem que a adoção do postponement exige não apenas a reconfiguração do sistema logístico, mas também mudanças no relacionamento entre os membros da cadeia de suprimentos, investimentos em tecnologia e visão estratégica de seus líderes. Novos pré-requisitos foram identificados, sugerindo que o atual modelo para implementação do postponement necessita ser ampliado. As empresas precisam investir em mudanças de produto, processo, relacionamento na cadeia de suprimento e organizacionais para usufruir dos benefícios do postponement.

Palavras-chave: postponement; supply chain management; logística.

\begin{abstract}
The purpose of this article is to investigate the adoption of postponement in the business environment in Brazil and which factors favor its implementation. This study used the methodology of multi-case studies to investigate this phenomenon that is well known in the literature, but rare in real business life. Results suggest that the implementation of postponement requires not only the reconfiguration of the logistics systems, but changes in the relationship between members in the supply chain, investments in technology and the strategic vision of their leaders. New prerequisites were identified, suggesting that the existing framework for implementation of postponement needs to be extended. Companies should invest in change of products, processes, supply chain relationships and organization to enjoy the benefits of postponement.
\end{abstract}

Key words: postponement; supply chain management; logistics. 


\section{INTRODUÇÃO}

Um dos novos desafios dos profissionais de operações e supply chain nos tempos atuais é reconfigurar a cadeia de suprimento (Boone, Craighead, \& Hanna, 2007), para responder rapidamente à demanda de produto com características cada vez mais inovadoras (Fisher, 1997; Randall \& Ulrich, 2001). Mudanças importantes de produtos, processos, e cadeias de suprimentos serão necessárias (Fine, 1998) para atender às demandas cada vez mais personalizadas dos consumidores.

A Dell é exemplo clássico de cadeia de suprimento responsiva, pois é uma empresa que fornece produtos personalizados em poucos dias, a preços compatíveis com um sistema de produção em massa (Fisher, 1997). A montadora General Motors Brasil [GMB] segue o mesmo caminho, atende a $70 \%$ da demanda dos veículos marca Celta pela internet e os entrega entre 4 e 10 dias aos consumidores, a partir de quatro centros de distribuição (Csillag \& Sampaio, 2002). A indústria de tintas foi a pioneira neste processo responsivo, pois permite a mistura de tintas nas lojas de material de construção, chamado de sistema selfcolor, que possibilita a escolha ao consumidor de uma entre 8.000 alternativas de tonalidades de tintas.

O que existe em comum nos exemplos citados acima? A adoção do postponement, que consiste em uma estratégia de adiar a configuração final de produtos e/ou serviços o máximo possível dentro da cadeia de suprimento, em casos extremos transferindo etapas finais de manufatura para o canal de distribuição e/ou até para o próprio consumidor.

A prática de postponement é conhecida na literatura desde os anos 50 (Alderson, 1950); entretanto foi somente nesta última década que o tema despertou o interesse de executivos e acadêmicos (Yang, Yang, \& Wijngaard, 2005). Pouco ainda se sabe sobre os direcionadores e obstáculos de sua adoção. Apesar de notícias de alguns casos de sucesso no mundo empresarial, como Benetton, Whirlpool e Hewlett Packard, a realidade é que a adoção do princípio do postponement ainda se encontra pouco difundida, contrariando a previsão otimista de diversos autores (Battezzati \& Magnani, 2000; Brown, Lee, \& Petrakian, 2000; Dröge, Germain, \& Spears, 1995; Yang, Burns, \& Backhouse, 2004a).

Este artigo tem o propósito de investigar a prática de postponement no Brasil para entender: Por que empresas brasileiras adotaram estratégias de postponement somente nesta última década, considerando que o conceito é conhecido na literatura desde a década de 50? Quais os direcionadores na adoção dos princípios de postponement em empresas brasileiras?

Como a implementação do postponement ainda se encontra pouco difundida, com casos esporádicos de sucesso no Brasil e no mundo (Hoek, 2001; Yang \& Burns 2003; Yang et al., 2004a) respostas a estas questões são relevantes. A realização do estudo ocorreu em virtude da emergência no mercado nacional de importantes empresas que adotam tais princípios, sem que maiores esforços acadêmicos fossem realizados até o momento para entendimento deste fenômeno.

Para atingir os objetivos, o artigo está estruturado em cinco partes, contando com a introdução. A segunda parte realizou uma breve revisão da literatura. A metodologia de pesquisa é discutida na parte três. A quarta parte relata os resultados obtidos da pesquisa e apresenta a análise cruzada dos casos em face das questões propostas no estudo. A quinta parte faz uma síntese do estudo, e relata as limitações e lacunas para pesquisas futuras.

\section{REVISÃO DA LITERATURA}

As primeiras experiências empresariais que utilizam o princípio do postponement remontam à década de 20 (Knight, 1921). Na literatura a teoria foi elaborada por Alderson (1950) e mais tarde expandida por Bucklin (1965). Estes autores argumentaram que os custos relacionados a riscos e 
incertezas estão intrinsecamente ligados à intensidade de diferenciação espaciotemporal que ocorre durante os processos de manufatura e logística. Eles afirmaram que quanto maior o grau de adiamento da configuração final dos produtos, ao longo da cadeia de suprimento, menor a possibilidade de perdas e maior o nível de serviço prestado ao consumidor final.

Bucklin (1965) interpretou o postponement como sistema de divisão de riscos e benefícios entre todos os membros da cadeia de suprimento no atendimento das necessidades individuais dos consumidores. Alderson (1957) argumentou que o sucesso do serviço de alimentação self-service, lançado naquela época, era claro sinal de que a diferenciação nas fases iniciais do processo deveria ser evitada, e que algumas etapas finais deveriam até ser transferidas para o próprio consumidor.

Simultaneamente na área de operações, Starr (1965) dedicou-se a estudar o conceito de produção modular fundamentado na concepção de produtos complexos, a partir de subsistemas menores que são combinados de múltiplas formas durante o processo de manufatura ou montagem final, da mesma forma que as crianças montavam diferentes objetos a partir de blocos lego. O modelo modular de produção propõe a divisão do processo produtivo em duas etapas:

1) Processo de transformação primário: transforma matérias-primas e insumos em módulos ou componentes e;

2) Processo de montagem final: combina os diferentes módulos no máximo número possível de produtos acabados.

Os resultados esperados seriam reduções de investimentos em pesquisa, em custos operacionais, em custos logísticos e melhoria dos serviços prestados aos clientes.

Naquela época estes autores reconheceram que a demanda crescente pela personalização não seria satisfeita pelo sistema de produção em massa vigente. Seria necessário o envolvimento das áreas de operações e logística, para maximizar a possibilidade de diferenciação. Apesar de reveladores, os trabalhos de Alderson (1950), Bucklin (1965) e Starr (1965) não despertaram a merecida atenção dos executivos, em função do predomínio da mentalidade de produção em massa no mercado.

Zinn e Bowersox (1988) retomaram o estudo do tema no final da década de 80, argumentando que o postponement é uma eficaz estratégia para aprimorar os sistemas de distribuição. Os autores identificaram cinco tipos de estratégias de postponement, quatro relacionadas com alterações de forma do produto (etiquetagem, embalagem, montagem e manufatura) e o quinto relacionada ao tempo (centralização dos estoques). Utilizando modelos de simulação, os autores comprovaram a existência de situações em que a adoção do postponement é justificada economicamente.

Para Bowersox e Closs (1996) existem dois tipos de postponement que são críticos: postponement de produção (forma) e postponement da Logística (lugar). Os dois tipos de postponement reduzem os riscos, mas de maneiras diferentes. O postponement de produção concentra-se na forma do produto, movimentando itens inacabados ao longo da rede logística, visando à sua consolidação e montagem antes da entrega ao consumidor. O postponement de logística tem como foco o tempo, propõe a estocagem de produtos acabados em local central, para responder rapidamente ao pedido recebido do cliente.

Na década de 90, Christopher (1992) ampliou novamente o escopo do conceito, posicionando o postponement como estratégia eficaz e capaz de reorganizar redes de suprimentos globais. $\mathrm{O}$ autor recomendou um cuidadoso exame de toda cadeia de valor à procura de oportunidades para 'adiar' a configuração final do produto, isto é, incentivar o despacho de componentes e embalagens intermediárias em redes de suprimentos globais para montagem final nos países de destino.

Cooper (1993) também identificou diferentes tipos de postponement em mercados globalizados, categorizados como: estratégia de manufatura descentralizada, manufatura centralizada, postponement de montagem e postponement de embalagem. 
Pagh e Cooper (1998, p. 18) fizeram importante contribuição ao estabelecer nova morfologia, ao combinar os conceitos de postponement e especulação

1) Estratégia de especulação plena: todas as operações são concretizadas o mais cedo possível na cadeia de suprimento e orientadas por previsões de demanda.

2) Estratégia de postponement de manufatura: algumas operações, tais como pequenas montagens, embalagem ou fixação de rótulos, são realizadas dentro dos canais de distribuição.

3) Estratégia de postponement de logística: o planejamento da produção é especulativo, porém a distribuição é adiada. Os pedidos dos clientes são atendidos a partir de estoques centralizados.

4) Estratégia de postponement pleno: representa o maior nível possível de postponement; consiste em somente realizar as operações de manufatura e logística contra o pedido do consumidor final.

Waller, Dabholker e Gentry, (2000); Brown et al. (2000); Yang e Burns (2003) e Yang et al. (2004b) propõem novas categorias de postponement, expandindo o leque de possibilidade.

Resumindo, existem várias tipologias de postponement, envolvendo múltiplos elementos e diferentes aspectos. Isto é natural, uma vez que existem várias combinações possíveis de adiamento espaciotemporal e de forma no mundo empresarial. A Tabela 1 apresenta as diferentes tipologias do conceito de postponement dos diversos autores discutidos neste artigo.

Tabela 1

Tipos de Postponement

\begin{tabular}{ll}
\hline Autor(es) & Classificação das estratégias de postponement \\
\hline Alderson (1950) & Postponement \\
Bucklin (1965) & Especulação, Postponement \\
Zinn e Bowersox (1988) & $\begin{array}{l}\text { Postponement forma (Etiquetagem, Embalagem Montagem e } \\
\text { Manufatura) e postponement de tempo }\end{array}$ \\
Cooper (1993) & $\begin{array}{l}\text { Manufatura Descentralizada, Centralizada, Postponement de Montagem } \\
\text { e embalagem }\end{array}$ \\
Bowersox e Closs (1996) & $\begin{array}{l}\text { Postponement de logística (combinação de tempo e lugar) e } \\
\text { postponement forma (manufatura) }\end{array}$ \\
Pagh e Cooper (1998) & $\begin{array}{l}\text { Especulação plena, postponement de Logística, postponement de } \\
\text { manufatura e postponement pleno }\end{array}$ \\
Waller et al. (2000) & $\begin{array}{l}\text { Postponement de produção, postponement a montante e postponement a } \\
\text { jusante }\end{array}$ \\
Postponement de produto e postponement de processo. \\
Yang e Burns (2003)
\end{tabular}

\section{Direcionadores da Adoção do Postponement na Literatura}

Os casos estudados até o momento na literatura indicam a necessidade da presença de alguns direcionadores de mercado, de produtos e de processo para a adoção do postponement. A Tabela 2 resume os principais fatores encontrados na literatura. 
Tabela 2

\section{Direcionadores que Influenciam a adoção do Postponement}

\begin{tabular}{|c|c|}
\hline Autor(es) & Direcionadores \\
\hline Bowersox e Closs (1996) & $\begin{array}{l}\text { Preço elevado do produto; demanda instável, elevado número de } \\
\text { marcas e versões do produto, variações no peso e tamanho do produto e } \\
\text { alto percentual de materiais ubíquos. }\end{array}$ \\
\hline Cooper (1993) & $\begin{array}{l}\text { Processo modular; baixa complexidade na operação de personalização; } \\
\text { projeto modular do produto; fontes de múltiplas locações; módulos } \\
\text { intercambiáveis; formulação específica do produto; periféricos } \\
\text { específicos; produto de alto valor monetário; aumento do peso ou } \\
\text { volume; ciclo de vida do produto; elevada oscilação da demanda; } \\
\text { necessidade de lead time curto e confiável; competição em preço; } \\
\text { mercado segmentado. }\end{array}$ \\
\hline Dröge, et al. (1995) & Tecnologia da informação; baixa previsibilidade da demanda. \\
\hline Hoek (1998) & $\begin{array}{l}\text { Intensidade de utilização da tecnologia de informação integrando os } \\
\text { processos internos; intensidade de utilização da tecnologia da } \\
\text { informação, integrando os processos interorganizacionais; nível de } \\
\text { turbulência do mercado; freqüência de mudança tecnológica de } \\
\text { produtos e processos; nível de complexidade da etapa final do processo } \\
\text { de manufatura; grau de modularidade e padronização do produto; nível } \\
\text { de diferenciação possível durante o estágio final do processo de } \\
\text { manufatura e número de atividades de customização realizada pela } \\
\text { operação. }\end{array}$ \\
\hline Pagh e Cooper (1998) & $\begin{array}{l}\text { Estágio do ciclo de vida; volume; estratégia de custo/serviço; tipo de } \\
\text { produto; variedade; perfil de valor; densidade monetária; tempo de } \\
\text { entrega; freqüência de entrega; nível de instabilidade da demanda; } \\
\text { economia de escala; complexidade da personalização. }\end{array}$ \\
\hline Brown et al. (2000) & $\begin{array}{l}\text { Tempo de manufatura; variedade de produto; tempo de produção e } \\
\text { incerteza da demanda }\end{array}$ \\
\hline Waller et al. (2000) & Personalização de produto e velocidade de produção \\
\hline Yang e Burns (2003) & $\begin{array}{l}\text { Posição do ponto de desacoplamento; integração da cadeia de } \\
\text { suprimento e controle da cadeia de suprimento }\end{array}$ \\
\hline Yang et al. (2004a) & $\begin{array}{l}\text { Incerteza da demanda; variabilidade da demanda; tecnologia da } \\
\text { informação disponível }\end{array}$ \\
\hline Yang et al. (2004b) & Grau de incerteza e modularidade \\
\hline Krajewski, Wei e Tang (2005) & $\begin{array}{l}\text { Magnitude e freqüência das mudanças permitidas; acuracidade do } \\
\text { momento do transporte e intervalo entre revisões de planejamento. }\end{array}$ \\
\hline Huang e Li (2008) & Incerteza do ambiente, fatores gerenciais, maturidade da T.I. \\
\hline
\end{tabular}

Uma análise dos direcionadores da Tabela 2 indica a predominância de pré-requisitos técnicos associados a produto, processo e mercado para a adoção dos diversos tipos de postponement. Duas regras gerais podem ser aplicadas a todos os tipos de postponement:

1) As oportunidades de adoção surgem à medida que os erros de previsão de demanda são elevados, causando aumento nos custos de marketing e distribuição.

2) As oportunidades de adoção são maiores para item de elevado valor agregado, pois neste caso temos maior custo de armazenagem. 
A decisão do tipo de postponement depende do grau de modalidade de produto, processos e cadeia de suprimento (Chiou, Wu, \& Hsu, 2002; Fine, 1998; Hoek, 1998; Ulrich, 1995; Yang et al., 2004a). Por exemplo, Yang e Burns (2003) desenvolveram um modelo conceitual para decisão do tipo de postponement em função dos direcionadores: grau de modularidade e nível de incerteza.

Esta pesquisa teve entre seus objetivos verificar a presença dos direcionadores listados na Tabela $2 \mathrm{e}$ identificar a emergência de novos direcionadores que expliquem a adoção do postponement no ambiente de negócios brasileiro.

\section{Metodologia de Pesquisa}

O propósito da pesquisa foi investigar um fenômeno emergente, a adoção do postponement em empresas brasileiras, evento contemporâneo sobre o qual o pesquisador não tem nenhum controle. Neste contexto, a metodologia indicada por diversos autores (Eisenhardt, 1989; Ellram, 1996; Yin, 2003; entre outros) é o estudo de caso. O conhecimento disponível sobre o fenômeno de interesse é escasso, especialmente na literatura de um país em desenvolvimento como o Brasil. Neste caso, a pesquisa exploratória é essencial para desenvolver e prover novas idéias e descobertas.

O método de estudo de caso (Yin, 2003) utiliza uma teoria previamente desenvolvida como modelo com o qual se devem comparar os resultados empíricos do estudo de caso. Se dois ou mais casos utilizados sustentam a teoria, pode-se assim solicitar uma replicação. Para entender a implementação de postponement em diferentes contextos, esta pesquisa optou pelo modelo de estudo de casos múltiplos para aumentar o grau de validade externa e também reduzir qualquer viés do pesquisador no decorrer da pesquisa.

Como é coerente na metodologia de estudo de caso, não tem sentido estabelecer hipóteses formais a priori. Entretanto, conforme Yin (2003), é satisfatório o estabelecimento de proposições, como respostas às questões de estudo já apresentadas na introdução. Este procedimento ajuda o pesquisador a nortear o trabalho de coleta de informações.

Proposição 1 - A adoção dos princípios de postponement só se justifica em situações em que ocorre pressão de agentes externos à organização, como, por exemplo, mudanças da legislação, pressão de fornecedores e mudanças no comportamento do consumidor, que ocorreram mais nesta última década. No Brasil os executivos não foram proativos na sua implementação. Este fato levou à implementação de um modo descoordenado, sem explorar todas as potencialidades de ganho possíveis com o postponement.

Proposição 2 - Os direcionadores da literatura que explicam a adoção do postponement não são suficientes para explicar a difusão do postponement no Brasil, novos direcionadores relevantes serão encontrados.

\section{Unidade de Análise}

A unidade de análise para o presente estudo de caso foi a empresa do setor industrial brasileiro que adota um ou mais tipos de postponement identificados na literatura. O nível de análise considerado seguiu a opinião de seus tomadores de decisão: executivos, diretores e/ou gerentes.

\section{Seleção dos Casos}

A presente pesquisa foi composta arbitrariamente por uma amostra de 5 empresas, devido ao restrito número de casos conhecidos que utilizam a estratégia de postponement. O objetivo não foi fazer generalizações estatísticas de resultados, mas somente generalizações analíticas. 
Foram selecionadas empresas com poucas características e elementos em comum, uma vez que o objetivo foi entender o processo de implementação de postponement em diferentes contextos. Os casos selecionados possuem produtos e/ou serviços em diferentes estágios de seu ciclo de vida e adiam uma quantidade diferente de atividades de manufatura e/ou distribuição, antes de receber o pedido final de seus clientes, conforme mostra a Figura 1. Isto significa que a pesquisa avaliou uma ampla gama de tipos possíveis de postponement mencionados na literatura; porém tem a desvantagem de estudar apenas um ou dois casos de cada categoria.

\begin{tabular}{|c|c|c|c|}
\hline $\begin{array}{l}\text { Postponement } \\
\text { pleno }\end{array}$ & $\begin{array}{l}\text { Fabricante de } \\
\text { Caminhões }\end{array}$ & $\begin{array}{l}\text { Fabricante de } \\
\text { Computador }\end{array}$ & \\
\hline $\begin{array}{l}\text { Postponement } \\
\text { logística }\end{array}$ & $\begin{array}{l}\text { Fabricante de } \\
\text { Automóveis }\end{array}$ & & \\
\hline \multirow[t]{2}{*}{$\begin{array}{l}\text { Postponement } \\
\text { Manufatura }\end{array}$} & & $\begin{array}{c}\text { Fabricante de Tintas } \\
\text { Decorativa }\end{array}$ & $\begin{array}{c}\text { Fabricante de } \\
\text { Bolos }\end{array}$ \\
\hline & $\begin{array}{l}\text { introdução } \\
\text { crescimento }\end{array}$ & $\begin{array}{l}\text { crescimento } \\
\text { maturidade }\end{array}$ & $\begin{array}{l}\text { maturidade } \\
\text { declínio }\end{array}$ \\
\hline
\end{tabular}

Figura 1: Postponement versus Ciclo de Vida

Fonte: elaborado pelos autores.

\section{Protocolo de Coleta de Dados}

Vários autores (Eisenhardt, 1989; Ellram, 1996; Yin, 2003) argumentam que a coleta de dados qualitativos deve ser baseada em múltiplas fontes de evidência, mas sempre convergindo ao mesmo conjunto de fatos e descobertas. Dados qualitativos são considerados evidências da ligação entre dados e proposições. Os estudos de caso foram realizados sempre envolvendo entrevistas em profundidade de dois ou mais profissionais da empresa, conforme mostra a Tabela 3. Normalmente foram realizadas duas ou mais visitas à empresa pesquisada e a alguns dos seus membros do canal de distribuição, em encontros nunca inferiores a 3 horas de trabalho.

Tabela 3

Relação de Entrevistados

\begin{tabular}{llll}
\hline Caso & Indústria & Produto & Funções dos entrevistados \\
\hline GMB & Automotiva & Carro & Gerente de comércio eletrônico \\
& & Gerente de Fábrica \\
& & Gerente de Relações Institucionais \\
& & Gerente do Operador Logístico \\
VW & Automotiva & Caminhões e ônibus & Diretor de Manufatura \\
& & Gerente de Recursos Humanos \\
& & Gerente de Logística \\
& & Gerente de Desenvolvimento de Produtos \\
& & Gerente de Qualidade \\
& & Gerente do módulo de Power Train \\
\hline
\end{tabular}

Continua 
Tabela 3 (continuação)

\begin{tabular}{llll}
\hline Caso & Indústria & Produto & Funções dos entrevistados \\
\hline DC & Eletrônica & Computadores e servidores & $\begin{array}{l}\text { Gerente de Logística } \\
\text { Gerente de Relações Institucionais }\end{array}$ \\
& & Tintas decorativas & $\begin{array}{l}\text { Gerente de Logística } \\
\text { BA }\end{array}$ \\
& Tintas & Gerente de marketing \\
AP & Alimentos & Bolos e confeitos & $\begin{array}{l}\text { Gerente de Produção } \\
\text { Gerente de uma Loja de Franchising }\end{array}$ \\
\hline
\end{tabular}

Nota. Fonte: elaborado pelos autores.

A principal fonte de dados foram as entrevistas realizadas com executivos e profissionais das áreas logísticas, operações e marketing em diferentes níveis da estrutura organizacional. Também foram coletadas informações de registros de arquivos, como mapas, índices de desempenho, registros financeiros, informações de mercado, entre outros. Finalmente foram realizadas observações diretas, ao longo da visita de campo, da atividade de postponement praticada pela organização.

Yin (2003) alerta aos pesquisadores que é importante ter uma estratégia analítica para coleta das evidências de um estudo de caso, antes de iniciar quaisquer trabalhos de campo. A estrutura de análise dos casos da pesquisa foi a seguinte: primeiramente realizar uma narrativa simples sobre cada um dos casos individualmente. Em seguida foi dedicada uma seção para mostrar e analisar os resultados dos casos cruzados. Foi estruturada uma matriz de variáveis importantes para ser observada durante a condução das entrevistas dos executivos e profissionais das empresas pesquisadas. Estas variáveis foram escolhidas, considerando as perguntas da pesquisa e a revisão da literatura.

\section{Resultados DA PESQUisa}

\section{Análise Individual dos Casos}

Os estudos de casos realizados podem ser identificados pelos seguintes códigos:

1) GMB, uma montadora de veículos.

2) VW, uma montadora de caminhões e ônibus.

3) DC, uma fabricante de computadores pessoais.

4) BA, uma fabricante de tintas para decoração.

5) AP, uma fabricante de bolos e confeitos.

A GMB é uma unidade de negócio da empresa General Motors do Brasil, mais especificamente é o Complexo Industrial de Gravataí. A fábrica foi inaugurada em julho de 2000 e chamou atenção, tanto de acadêmicos quanto de executivos, não só no Brasil, mas em todo o mundo (Csillag \& Sampaio, 2002). A idéia central da planta era vender um carro montado sob medida para o consumidor final. Este projeto foi revolucionário no mundo automotivo porque os veículos eram vendidos pela internet, o preço era único e o tempo médio de entrega, relativamente baixo (4 dias). Os fornecedores foram responsáveis por fornecimento de módulos, não peças, além de serem co-investidores do empreendimento. 
A VW é a Volkswagen Caminhões e Ônibus de Resende-RJ, muito conhecida no mundo empresarial e acadêmico pelo sucesso da adoção do conceito de consórcio modular (Corrêa, 2001). Diferente de qualquer outra planta automotiva do mundo, esta fábrica possui seus principais fornecedores dentro da linha de montagem que adiciona seus componentes diretamente nos veículos. A empresa investiu no conceito de postponement, denominado internamente de caminhão Taylor Made, para entender e atender melhor as necessidades dos clientes.

A DC é um importante fabricante de computadores. A empresa utiliza o modelo de vendas diretas de computadores e servidores, seja pela Web, por telefone e por intermédio de gerente de conta. A empresa entrega seus produtos montados sob medida em todo o território nacional. O resultado é a redução de estoques ao longo de toda a cadeia de suprimento. Trata-se da aplicação do conceito de postponement de montagem, uma vez que todo o processo de manufatura só é iniciado após o cliente, corporativo ou usuário doméstico, colocar seu pedido.

A BA é uma unidade de negócio de tintas imobiliárias do grupo Basf, localizada em São Bernardo do Campo (SP). As três principais marcas da companhia são a Suvinil, a Novinil e a Selavinil. A marca Suvinil introduziu em 1992 o sistema tintométrico Self color, foco de interesse deste trabalho. Trata-se de uma das primeiras aplicações de postponement no mercado brasileiro.

A empresa AP é a Rede Amor aos Pedaços, especializada na fabricação de bolos e confeitos, chegou ao mercado em 1982 com uma novidade: o cliente tem agora a opção de escolher o sabor e tamanhos dos seus bolos e confeitos artesanais. Neste caso, a empresa utiliza a modalidade de postponement de manufatura, ou seja, mantêm seus produtos em estado neutro como módulos intercambiáveis, o que permite maior poder de customização. A tarefa de montagem final dos bolos e doces é delegada aos funcionários do canal de distribuição, mais próximos dos clientes e, portanto, mais aptos para conhecer aquilo que realmente os clientes desejam. Este estilo diferenciado e inovador faz da rede a maior doçaria da América Latina, com mais de 40 lojas nos Estados de São Paulo e Rio de Janeiro.

\section{Análise Cruzada dos Casos}

Durante as entrevistas foi investigado o processo utilizado pela empresa para atender à demanda do mercado, podendo seu processo oscilar entre especulação até um dos diferentes tipos de postponement. A Tabela 4 apresenta os resultados encontrados.

Tabela 4

\section{Resposta à Demanda das Empresas Pesquisadas}

\section{Resposta à Demanda (em percentual da demanda)}

Especulação

Postponement Pleno

Postponement de Logística

Postponement de Manufatura

\section{Atividade adiada}

Projeto de engenharia

Operações de Manufatura

Montagem final

Logística

Embalagem e/ou etiquetagem

Manufatura final no canal

\begin{tabular}{lllll}
\hline GM & VW & DC & BA & AP \\
\hline
\end{tabular}

$\begin{array}{lllll}30 \% & 40 \% & & 80 \% & \\ 10 \% & 60 \% & 100 \% & & \\ 60 \% & & & & \\ 60 \% & & & 20 \% & 100 \%\end{array}$

$10 \%$

$10 \% \quad 50 \% \quad 100 \%$

$60 \%$

$60 \%$

$20 \% \quad 100 \%$ 
O resultado da Tabela 4 indica que diferentes modalidades de postponement podem ser adotadas por uma mesma organização; como exemplo, o caso da GM, que implementou o conceito de postponement de logística, uma vez que os veículos são mantidos em centros de distribuição e são deslocados diretamente para o consumidor somente após receber o pedido. Porém ela também executa uma série de pequenas montagens de itens de personalização nas concessionárias, o que caracteriza a adoção de uma política de postponement de manufatura. Esta ocorrência foi pouco mencionada na literatura; somente Rabinovich e Evers (2003) e Tibben-Lembke e Bassok, (2005) mencionaram a ocorrência de múltiplos tipos de postponement em uma única cadeia de suprimento.

\section{Pergunta n 1}

1) Por que empresas brasileiras adotaram estratégias de postponement somente nesta última década, considerando que o conceito é conhecido na literatura desde a década de 50?

Proposição 1 - A adoção dos princípios de postponement só se justifica em situações em que ocorre pressão de agentes externos à organização, como, por exemplo, mudanças da legislação, pressão de fornecedores e mudanças no comportamento do consumidor, que ocorreram mais nesta última década. No Brasil os executivos não foram proativos na sua implementação. Este fato levou à implementação de um modo descoordenado, sem explorar todas as potencialidades de ganho possíveis com o postponement. (Proposição Rejeitada).

De fato, alguns entrevistados confirmaram que os agentes externos foram determinantes para a tomada de decisão gerencial, como, por exemplo, temos o explicitado a seguir.

1) A General Motors decidiu-se pela adoção do sistema de venda direta pela Internet somente após o governo FHC decretar um aumento dos impostos PIS/Cofins. A idéia de venda direta ao consumidor surgiu em 1999, resultado do trabalho de um grupo gerencial encarregado do projeto de redução dos custos de distribuição do veículo Celta, que logo seria lançado no mercado. A atitude da montadora foi mais reativa a uma mudança de impostos do que tomadora de uma ação proativa, fruto de uma visão estratégica na época do projeto do Complexo Industrial de Gravataí em 1996.

2) A Indústria de Tintas foi pressionada pelos fabricantes norte-americanos de máquinas do sistema selfcolor a adotar o conceito no Brasil, visando à venda de seus equipamentos, uma vez que o mercado norte-americano vende $80 \%$ de sua produção por tal sistema. A mudança foi também reativa e pouco planejada.

No entanto não se pode afirmar que a lógica do postponement só se aplica quando ocorre o estímulo de fontes externas. Na VW, a DC e a AP a adoção foi resultado de uma mudança estratégica definida pela liderança. Estas empresas já nasceram sob os princípios do postponement.

Segundo os entrevistados, as seguintes razões para a adoção tardia do postponement foram:

“Após a abertura do país ao mercado internacional, a competitividade aumentou muito e o consumidor passou a ter maior poder de escolha de seus fornecedores. Sem este aumento de concorrência, investimentos em flexibilidade não seriam necessários e até poderiam não se justificar" (Diretor de Manufatura, VW).

"O desenvolvimento da tecnologia da informação e telecomunicações possibilitou o estabelecimento de novas formas de relacionamentos entre fabricantes e consumidores, muito mais próximas e sem intermediários, mesmo em situações com um elevado volume de transações" (Gerente de Comércio Eletrônico, GMB).

\section{$\operatorname{Pergunta~}^{0} 2$}

2) Quais os direcionadores na adoção dos princípios de postponement em empresas brasileiras? 
Proposição 2 - Os direcionadores da literatura que explicam a difusão do postponement não são suficientes para explicar a adoção do postponement no Brasil, novos direcionadores relevantes serão encontrados. (Proposição Confirmada).

Há características operacionais relevantes cuja presença é considerada necessária para possibilitar a adoção do postponement. Todas estas características foram avaliadas para cada caso estudado, conforme mostra a Tabela 5.

Tabela 5

Direcionadores que Favorecem a Adoção de Postponement

\begin{tabular}{|c|c|c|c|c|c|}
\hline \multirow{3}{*}{ CARACTERÍSTICAS } & \multicolumn{5}{|c|}{ POSTPONEMENT } \\
\hline & \multicolumn{2}{|c|}{ PLENO } & \multirow{2}{*}{$\begin{array}{c}\text { LOGÍSTICA } \\
\text { GM }\end{array}$} & \multicolumn{2}{|c|}{ MANUFATURA } \\
\hline & DC & VW & & BA & $\mathbf{A P}$ \\
\hline \multicolumn{6}{|l|}{ PRODUTO } \\
\hline Produto Modular & Sim & Sim & Parcialmente & Sim & Sim \\
\hline Formulação específica do produto & Elevada & Elevada & Moderada & Elevada & Elevada \\
\hline Periféricos específicos & Sim & Sim & Sim & Sim & Sim \\
\hline Densidade monetária & Elevada & Elevada & Elevada & Moderada & Moderada \\
\hline Variação de peso do produto & Não & Não & Não & Não & Não \\
\hline Variação do volume do produto & Não & Não & Não & Sim & Sim \\
\hline Peso dos Materiais Ubíquos & Não & Não & Não & Não & Não \\
\hline $\begin{array}{l}\text { Variação no Tamanho da } \\
\text { Embalagem }\end{array}$ & Não & Não & Não & Não & Não \\
\hline Intensidade de personalização & Elevada & Elevada & Moderada & Elevada & Elevada \\
\hline \multicolumn{6}{|l|}{ PROCESSO } \\
\hline Viabilidade de desacoplamento & Não & Parcialmente & Parcialmente & Sim & Sim \\
\hline Complexidade da customização & Elevada & Elevada & Elevada & Baixa & Baixa \\
\hline Projeto modular do processo & Sim & Sim & Sim & Sim & Sim \\
\hline Fontes de múltiplas locações & Sim & Sim & Sim & Sim & Sim \\
\hline Economias de Escala & Sim & Sim & Sim & Sim & Sim \\
\hline \multicolumn{6}{|l|}{ MERCADO } \\
\hline Estágio do Ciclo de Vida & Maturação & Maturação & Maturação & Maturação & Maturação \\
\hline Ciclo de vida & 6 meses & 2 a 3 anos & 2 a 3 anos & $\begin{array}{l}\text { Curto- } \\
\text { lançamento }\end{array}$ & $\begin{array}{l}\text { Curto- } \\
\text { lançamento }\end{array}$ \\
\hline Tempo de Entrega & 3 a 7 dias & 45 dias & 4 a 10 dias & $15 \mathrm{~min}$. & $15 \mathrm{~min}$. \\
\hline Freqüência de Entrega & Baixa & Baixa & Baixa & Media & Media \\
\hline Volume de vendas & Elevado & Baixo & Elevado & Elevado & Elevado \\
\hline Incerteza da demanda & Elevada & Elevada & Elevada & Elevada & Elevada \\
\hline Competição em preço/serviço & Sim & Sim & Sim & Limitado & Limitado \\
\hline Mercado Segmentado & Sim & Sim & Sim & Sim & Sim \\
\hline
\end{tabular}

Nota. $\square$ Resultado conflitante com a teoria.

Fonte: elaborado pelos autores. 
O caso DC tem todas as características operacionais que favorecem a implementação da modalidade do postponement pleno, que permite realizar suas operações de manufatura e logística após o pedido dos clientes. Realmente não tem muito sentido montar computadores com configurações de última geração, sem ter a mínima certeza de atender aos requisitos dos consumidores, assumindo todo o risco de obsolescência do produto nos canais de distribuição e vendas. A presença destas características operacionais de produto, processo e mercado foram importantes, mas não suficientes para explicar a adoção do postponement, pois ainda temos outras empresas fabricantes de computadores no mercado que adotam estratégias especulativas de manufatura e distribuição.

$\mathrm{O}$ caso VW também tem todas as características favoráveis à prática do postponement, sendo a modalidade postponement pleno a alternativa estratégica mais adequada, principalmente devido ao prazo de entrega de 45 dias praticado no mercado, possibilitando realizar o planejamento de manufatura e logística necessárias para atendimento das necessidades individuais de seus clientes. Cerca de $50 \%$ dos clientes são grandes empresas. No entanto, ainda há espaço para a prática simultânea da modalidade de postponement de manufatura, uma vez que alguns componentes são passíveis de serem desacoplados da montagem primária, como a distância entre eixos, possibilitando assim a realização de pequenas personalizações após o recebimento do pedido de clientes, principalmente para segmentos de mercados específicos como caminhões especiais.

O caso GM é interessante, pois a empresa pode até pensar em adotar a modalidade radical de postponement pleno, pois possui uma série de características operacionais favoráveis à adoção desta modalidade, conforme mostrado na Tabela 5. Entretanto, elevados volumes comercializados, a baixa flexibilidade de suas linhas de montagem e os pequenos prazos de entrega praticados no mercado são grandes obstáculos para a adoção desta modalidade. A empresa corria o risco de perdas de vendas, pois os prazos de entrega de seus veículos seriam elevados, acima de seus concorrentes. Uma opção seria ofertar uma ampla gama de possibilidades de personalização, suficiente para superar as expectativas do cliente no quesito qualidade e compensar a prática de prazo de entrega num nível superior à média da concorrência. Outro obstáculo é o restrito grau de modularidade dos veículos que impede qualquer desejo de personalização mais intensa, além da agregação dos acessórios normalmente ofertados pelas montadoras. A GM optou pela adoção da modalidade de postponement de logística, promovendo a centralização de seus estoques de veículos em centros de distribuição estrategicamente posicionados no mercado. Este posicionamento não significa que a empresa não possa modificar sua estratégia de resposta à demanda; tudo depende do nível de investimento da organização em modularidade e parcerias com fornecedores e revendas.

Os casos BA e a AP são semelhantes, possuem um conjunto de características operacionais que justificam a adoção da estratégia de postponement de manufatura. Trata-se de produtos modulares, que possuem processos que favorecem o desacoplamento das operações de manufatura em atividades primárias e complementares de baixa complexidade. A atividade de manufatura final passa a ser delegada ao membro mais próximo do consumidor, nestes casos, a loja de material de construção e a franquia.

O resultado da pesquisa indica que as características operacionais citadas na literatura foram também encontradas nos casos pesquisados no mercado brasileiro. Somente algumas características estiveram ausentes, como a variação de peso do produto, o peso dos materiais ubíquos e variação no tamanho da embalagem. Isto é decorrente do reduzido número de casos avaliados.

A análise dos casos individuais indica que mais aspectos têm influência para viabilização do postponement no Brasil. A Tabela 6 resume os principais aspectos observados. As principais dimensões adicionais envolvidas são: cadeia de suprimento, Organização e Tecnologia. 
Tabela 6

Direcionadores Adicionais para a Adoção de Postponement

\begin{tabular}{lllclc}
\hline \multirow{2}{*}{ CARACTERíSTICAS } & \multicolumn{5}{c}{ POSTPONEMENT } \\
\cline { 2 - 6 } & \multicolumn{2}{c}{ PLENO } & LOGÍSTICA & \multicolumn{2}{c}{ MANUFATURA } \\
\cline { 2 - 6 } DC & VW & GM & BA & AP \\
\hline CADEIA DE SUPRIMENTO & & & & & \\
Relacionamento Colaborativo & Sim & Sim & Sim & Sim & Sim \\
Resposta rápida dos fornecedores & Sim & Sim & Sim & Sim & Sim \\
Proximidade com fornecedores & Sim & Sim & Sim & Não & Não \\
Seqüenciamento de Peças & Sim & Sim & Sim & Não & Não \\
Mudança na Legislação & Não & Não & Sim & Não & Não \\
Divisão de Riscos e Benefícios & Sim & Sim & Sim & Sim & Sim \\
Organizacionais & & & & & \\
Estratégia da organização & Sim & Sim & Não & Não & Sim \\
Comprometimento da liderança & Sim & Sim & Sim & Não & Sim \\
Tecnologia & & & & & \\
Sistema de pagamento & Sim & Sim & Sim & Não & Não \\
Fornecedores de equipamentos & Sim & Sim & Sim & Sim & Sim \\
\hline
\end{tabular}

Nota. Fonte: elaborado pelos autores.

Foram encontrados nos casos estudados outros direcionadores relevantes, citados pelos entrevistados, como essenciais para a implementação do postponement e que não foram mencionados anteriormente na literatura, conforme mostra a Tabela 6 . Tais direcionadores foram:

1) Relacionamento na Cadeia de suprimento. O relacionamento entre membros da cadeia de suprimento precisa ser mais colaborativo e próximo. Mudanças constantes na operação exigem resposta rápida e personalizada dos fornecedores. Em alguns tipos de postponement é inevitável a proximidade física e a entrega de componentes personalizados e seqüenciados. Foi observada a divisão de riscos e benefícios para incentivar a parceria entre os membros da cadeia de suprimento. No caso específico da GMB, foi necessário até uma mudança na legislação para possibilitar a venda direta de veículos ao consumidor final. Os entrevistados declaram que a necessária modularidade do produto, contribui para acelerar o ritmo das inovações, uma vez que o conhecimento fica concentrado em pouco fornecedores.

2) Aspectos Organizacionais: A estratégia e liderança dos executivos foram fundamentais para a adoção da estratégia de postponement. Foi observado que os melhores resultados foram alcançados pelas empresas que já nasceram orientadas pelo conceito de postponement. As empresas que o adotaram acidentalmente ou devido a pressão de agentes externos, GMB e BA, não usufruíram do total benefício possível com o postponement.

3) Tecnologia - A dimensão tecnologia tem papel fundamental na adoção de alguns tipos de postponement, principalmente para estabelecer o contato com o consumidor final. Este aspecto, apesar de citado na literatura (Dröge et al., 1995; Hoek, 1998; Yang et al., 2004a), se restringia a tecnologia da informação e telecomunicações. Entretanto novos equipamentos também são 
importantes para permitir o desacoplamento dos processos, como observado no caso do sistema self color da Basf. Neste estudo a dimensão tecnologia foi estabelecida em um espectro mais amplo.

A Tabela 7 resume as principais características das empresas estudadas, as modificações ocorridas na rede de suprimento e os resultados alcançados, segundo depoimento dos entrevistados. 
Tabela 7

\section{Impacto do Postponement sobre a Cadeia de Suprimento das Empresas Pesquisadas}

\begin{tabular}{|c|c|c|c|c|c|}
\hline CASO & GM & VW & DC & $\mathbf{B A}$ & $\mathbf{A P}$ \\
\hline Indústria & Automotiva & Automotiva & Eletroeletrônica & Tintas & Alimentos \\
\hline Produto & Carros & Caminhões & Computadores & $\begin{array}{l}\text { Tinta do mercado } \\
\text { imobiliário }\end{array}$ & Bolos e confeitos \\
\hline Tipo & Postponement de & Postponement & Postponement & Postponement de & Postponement \\
\hline de postponement & Logística & Pleno & Pleno & Manufatura & Manufatura \\
\hline $\begin{array}{l}\text { Reconfiguração da } \\
\text { Rede de Suprimento }\end{array}$ & $\begin{array}{l}\text { Formação do } \\
\text { Condomínio industrial/ } \\
\text { Venda direta pela internet }\end{array}$ & $\begin{array}{c}\text { Formação de } \\
\text { Consórcio Modular/ } \\
\text { Caminhão sob medida }\end{array}$ & $\begin{array}{l}\text { Venda direta } \\
\text { Pela internet }\end{array}$ & $\begin{array}{l}\text { Sistema } \\
\text { self color }\end{array}$ & $\begin{array}{l}\text { Sistema de Bolos sob } \\
\text { encomenda }\end{array}$ \\
\hline \multicolumn{6}{|c|}{ MUDANÇAS OCORRIDAS NOS INTEGRANTES DA CADEIA DE SUPRIMENTO } \\
\hline Fornecedores & $\begin{array}{l}\text { Just In time com peças } \\
\text { seqüenciadas }\end{array}$ & $\begin{array}{l}\text { Realizam a montagem de } \\
\text { seus componentes } \\
\text { diretamente na linha de } \\
\text { montagem }\end{array}$ & $\begin{array}{l}\text { Supply Logistic Center } \\
\text { fornece os componentes } \\
\text { rapidamente conforme as } \\
\text { necessidades do mercado }\end{array}$ & $\begin{array}{c}\text { Entrega Just in time } \\
\text { Inserção dos fornecedores de } \\
\text { equipamentos tintométrico } \\
\text { na cadeia }\end{array}$ & Entrega Just in time \\
\hline Manufatura & $\begin{array}{c}\text { Centralização dos Estoques } \\
\text { Fábrica Flexível }\end{array}$ & $\begin{array}{l}\text { Montagem de caminhões } \\
\text { 'Taylor Made' }\end{array}$ & $\begin{array}{c}\text { Montagem de computadores } \\
\text { sob medida }\end{array}$ & Processo Estável & Processo Estável \\
\hline Canal de Distribuição & $\begin{array}{l}\text { Venda direta com } \\
\text { Participação das } \\
\text { Concessionárias }\end{array}$ & Venda Direta & $\begin{array}{l}\text { Venda direta com } \\
\text { Logística Terceirizada }\end{array}$ & $\begin{array}{l}\text { Participação da revenda no } \\
\text { processo de industrialização } \\
\text { das tintas }\end{array}$ & $\begin{array}{l}\text { Participação da franquia no } \\
\text { processo de industrialização } \\
\text { dos Bolos }\end{array}$ \\
\hline \multicolumn{6}{|c|}{ PRINCIPAIS BENEFÍCIOS IDENTIFICADOS } \\
\hline Integrantes da cadeia & $\begin{array}{l}\text { Redução dos custos } \\
\text { Melhoria do nível de serviço } \\
\text { Elevada Gama de produtos }\end{array}$ & $\begin{array}{l}\text { Elevada Gama de produtos } \\
\text { Melhoria do nível de serviço }\end{array}$ & $\begin{array}{l}\text { Elevada gama de produtos } \\
\text { Redução dos custos }\end{array}$ & $\begin{array}{l}\text { Redução dos custos } \\
\text { Melhoria do nível de serviço } \\
\text { Elevada Gama de produtos }\end{array}$ & $\begin{array}{l}\text { Redução dos custos } \\
\text { Melhoria do nível de serviço } \\
\text { Elevada Gama de produtos }\end{array}$ \\
\hline Mercado & $\begin{array}{l}\text { Crescimento } \\
\text { Market Share }\end{array}$ & $\begin{array}{l}\text { Crescimento } \\
\text { Market Share }\end{array}$ & $\begin{array}{l}\text { Crescimento } \\
\text { Market Share }\end{array}$ & $\begin{array}{c}\text { Crescimento } \\
\text { Market Share de } 20 \% \text { ao } \\
\text { ano }\end{array}$ & $\begin{array}{l}\text { Crescimento } \\
\text { Market Share }\end{array}$ \\
\hline
\end{tabular}

Nota. Fonte: elaborado pelos autores. 
Conforme pode ser observado na Tabela 7 , todas as empresas que implementaram o postponement tiveram aumento de market share em seus segmentos de mercado. Apesar de que a metodologia de estudo de casos não permite fazer generalizações estatísticas, pode-se pelo menos afirmar: a personalização permitida pela prática do postponement proporcionou uma vantagem competitiva para suas respectivas empresas, segundo o depoimento dos entrevistados.

\section{SÍNTESE E CONCLUSÕES}

Neste estudo, foi identificado que o conceito de postponement ganha relevância como alternativa estratégica em ambientes de negócios com demandas cada vez mais mutantes e imprevisíveis e que vários fatores influenciam o processo de decisão dos executivos de sua adoção. As entrevistas em profundidade com executivos de operações, supply chain e marketing revelaram que o postponement não é estratégia para todo tipo de organização; existe uma série de condições ambientais, mercadológicas e pré-requisitos operacionais, cuja presença é obrigatória para viabilizar sua adoção. A questão não é querer implementar o postponement, mas sim poder. A organização precisa ter condições mínimas de estrutura, infra-estrutura e relacionamento interorganizacional para sua implementação.

O estudo revelou que a adoção do postponement está associado ao grau de concorrência do mercado, ambientes mais competitivos são mais propícios à adoção do postponement. Outro fator importante é o recente desenvolvimento da tecnologia da informação e telecomunicações que tem possibilitado o estabelecimento de relacionamentos muito mais próximos entre fabricantes e consumidores, praticamente sem intermediários, mesmo quando a situação envolve elevado volume de transações. Isto justifica o sistema de venda direta pela Internet adotado pela GMB.

A adoção do postponement depende do grau de modularidade de seus produtos e processos e cadeia de suprimento; tais sistemas não são fáceis de serem projetados e operados. Para que o conceito de modularidade funcione é necessário o desenvolvimento de subsistemas semi-independentes, onde diferentes empresas ficam encarregadas de módulos separados e buscam garantir que a cadeia funcione adequadamente. Este conceito permite também acelerar as inovações, uma vez que o conhecimento de cada organização fica concentrado em partes do sistema. A questão fundamental é a obtenção da coordenação entre empresas da cadeia de suprimento por meio da formação de parcerias e divisão de benefícios e riscos.

Outros pontos importantes que surgiram nas entrevistas, em relação à adoção do postponement, foram os aspectos culturais e interorganizacionais envolvidos na implementação de estratégias de postponement. O postponement não depende só das condições técnicas operacionais. Também depende da transformação das relações entre empresas. Sua implementação implica, muitas vezes, delegar atividades de manufatura para outros membros a jusante na cadeia de suprimento. Neste contexto, é importante quebrar as barreiras organizacionais que normalmente impedem o estabelecimento de relacionamentos colaborativos.

As lições apreendidas nos casos estudados são de muita valia, por tratar-se de empresas de destaque no mercado nacional que estão apresentando resultados positivos e concretos com a reconfiguração de suas redes de suprimentos. Porém não há evidência clara de que o postponement represente tendência e prática dominante no mercado, pelo menos em curto prazo. Foi constatada a utilização reduzida dos conceitos de postponement que, pelo potencial, deveria estar muito mais difundida. Conforme afirma Fisher (1997), o projeto de uma rede de suprimento responsiva não é uma tarefa simples; entretanto a recompensa - uma vantagem competitiva notável - pode fazer tais esforços valerem a pena.

É importante citar algumas das limitações da pesquisa. Duas limitações são reconhecidas: a primeira 
é a impossibilidade de generalização estatística dos resultados, devido à utilização do método de estudo de casos. Em segundo lugar, a pesquisa avaliou vários tipos de postponement; porém tem a desvantagem de estudar apenas um ou dois casos de cada categoria. Reproduções deste estudo mais focados em apenas um tipo de postponement pode trazer contribuições adicionais.

\section{Pesquisas Futuras}

Alguns pontos merecem estudos futuros.

- Pesquisa em diferentes setores, como o automobilístico e eletroeletrônico, representa uma contribuição para a avaliação do processo de difusão das práticas de postponement no mercado brasileiro.

- Muitos autores têm estudado o impacto do postponement sobre os membros a jusante da cadeia de suprimento; porém poucos se dedicaram a investigar o impacto sobre os membros a montante. Um estudo relevante seria investigar este tema.

- Uma pesquisa quantitativa dos fatores direcionadores da implementação do postponement pode confirmar estatisticamente os direcionadores mencionados nesta pesquisa.

Artigo recebido em 30.03.2008. Aprovado em 25.11.2008.

\section{REFERÊNCIAS BIBLIOGRÁFICAS}

Alderson, W. (1950). Marketing efficiency and the principle of postponement. Cost and profit outlook, $3(4), 15-18$.

Alderson, W. (1957). Marketing behavior and executive action: a functionalist approach to marketing theory. The American Economic Review, 47(6), 1058-1060.

Battezzati, L., \& Magnani, R. (2000). Supply chains for FMCG and industrial products in Italy: practices and the advantages of postponement. International Journal of Physical Distribution \& Logistics Management, 30(5), 413-424.

Boone, C. A., Craighead, C. W., \& Hanna, J. B. (2007). Postponement: an evolving supply chain concept. International Journal of Physical Distribution \& Logistics Management, 37(8), 594-611.

Bowersox, D. J., \& Closs, D. J. (1996). Logistical management: the integrated supply chain process. New York: McGraw-Hill Companies.

Brown, A. O., Lee, H. L., \& Petrakian, R. (2000). Xilinx improves its semiconductor supply chain using product and process postponement. Interfaces, 30(4), 65-80.

Bucklin, L. P. (1965). Postponement, speculation and the structure of distribution channels. Journal of Marketing Research, 2(1), 26-31.

Chiou, J. S., Wu, L. Y., \& Hsu, J. C. (2002). The adoption of form manufacturing strategy in a global system: the case of Taiwanese information technology industry. Journal of Business Logistics, 23(1), 107-124.

Christopher, M. (1992). Logistics and supply chain management: strategies for reducing costs and improving services. London: Pitman Publishing. 
Cooper, J. C. (1993). Logistics strategies for global businesses. International journal of physical distribution \& logistics management, 23(4), 12-23.

Corrêa, H. L. (2001). Os modelos modulares de gestão de redes de suprimentos (Relatório de Pesquisa N²9/2001). São Paulo, SP, Escola de Administração de Empresas, Fundação Getulio Vargas.

Csillag, J. M., \& Sampaio, M. (2002, abril). Complexo industrial de Gravataí: um estudo de caso. Proceedings of the Annual Conference of the Production and Operations, San Francisco, CA, USA, 13.

Dröge, C. L., Germain, R. N., \& Spears, N. (1995, Summer). From postponement as a strategic initiative affecting organization design. Proceedings of the American Marketing Association Educators' Conference, Washington, DC, USA.

Eisenhardt, K. M. (1989). Building theories from case study researches. Academy of Management Review, 14(4), 532-550.

Ellram, L. M. (1996). The use of the case study method in logistics research. Journal of Business Logistics, 17(2), 93-138.

Fine, C. (1992). Clockspeed. Massachusetts, U.S.A.: Perseus Books.

Fisher, M. L. (1997). What is the right supply chain for your product? Harvard Business Review, 75(2), 105-116.

Hoek, R. I. van (1998). Postponed manufacturing in European supply chain: a triangular approach. Doctoral dissertation, Netherlands Geographical Studies, Utrecht, Nederland, Holland.

Hoek, R. I. van (2001). The rediscovery of postponement a literature review and directions for research. Journal of Operations Management, 19(2), 161-184.

Huang, Y., \& Li, S. (2008) Postponement application in greater China and its related determinants. Transportation Journal, 47(3), 5-21.

Knight, F. H. (1921). Risk, uncertainty and profit. Boston, MA: Hart, Schaffner \& Marx; Houghton Mifflin Company.

Krajewski, L., Wei, J. C., \& Tang, L. L. (2005). Responding to schedule changes in build-to-order supply chains. Journal of Operations Management, 23(5), 452-69.

Pagh, J. D., \& Cooper, M. C. (1998). Supply chain postponement and speculation strategies: how to choose the right strategy. Journal of business logistics, 19(2), 13-32.

Rabinovich, E., \& Evers, P. T. (2003). Postponement effects on inventory performance and the impact of information systems. International Journal of Logistics Management, 14(1), 33-48.

Randall, T., \& Ulrich, K. (2001). Product variety, supply chain structure, and firm performance: analysis of the US bicycle industry. Management Science, 47(12), 1588-1604.

Starr, M. K. (1965). Modular production a new concept. Harvard Business Review, 43(6), 131-142.

Tibben-Lembke, R., \& Bassok, Y. (2005). An inventory model for delayed customization: a hybrid approach. European Journal of Operational Research, 165(3), 748-764.

Ulrich, K. (1995). The role of product architecture in the manufacturing firm. Research Policy, 24(3), 419-440.

Waller, M. A., Dabholker, P. A., \& Gentry, J. J. (2000). Postponement, product customization, and market-oriented supply chain management. Journal of Business Logistics, 21(2), 133-159. 
Yang, B., \& Burns, N. D. (2003). Implications of postponement for the supply chain. International Journal of Production Research, 41(9), 2075-2090.

Yang, B., Burns, N. D., \& Backhouse, C. J. (2004b). Management of uncertainty through postponement. International Journal of Production Research, 42(6), 1049-1064.

Yang, B., Burns, N. D., \& Backhouse, C. J. (2004a). Postponement: a review and an integrated framework. International Journal of Operations \& Production Management, 24(5), 468-487.

Yang, B., Yang, Y., \& Wijngaard, J. (2005). Impact of postponement on transport: an environmental perspective. The International Journal of Logistics Management, 16(2), 192-204.

Yin, R. K. (2003). Case study research: design and methods (3a ed.). California: Sage Publications, Inc.

Zinn, W., \& Bowersox, D. J. (1988). Planning physical distribution with the principle of postponement. Journal of Business Logistics, 9(2), 117-136. 